\title{
Endoscopic Follow-up for the Remnant Stomach After Early Cancer Surgery: Reply
}

\author{
Isao Nozaki • Akira Kurita
}

Published online: 2 November 2010

(C) Société Internationale de Chirurgie 2010

We thank Dr. Katsios [1] for his interest in our article and for raising some important points. We agree that one has to balance the cost effectiveness of the intensity of the endoscopic follow-up with the likelihood of events, which is why, based on our results, we recommend examinations every 2-3 years [2]. Furthermore, as we mentioned in our article, the tumors $(n=26)$ in the remnant stomach were not recurrent tumors but metachronous secondary gastric cancers. All the tumors were resected curatively by gastrectomy or endoscopic mucosal resection, and the prognosis for these 26 patients was good following the resections [2]. The first choice for the treatment of curable gastric cancer is surgical resection and not chemotherapy. Since symptomatic advanced gastric remnant cancers are associated with unresectable $\mathrm{T} 4$ tumors and poor prognosis [3], periodic postoperative endoscopies are necessary to detect them at an asymptomatic early stage. Typically, secondary metachronous gastric cancer develops within 5-10 years after the primary cancer surgery [2, 4]. Therefore, only two to five endoscopic examinations are needed if you follow our recommendations [2]. Moreover, patients without any of risk factors may need fewer examinations.

Postoperative endoscopic examinations are not only for detecting secondary metachronous gastric cancer or recurrent tumor but are also for detecting postoperative morbidities after gastrectomy, such as peptic ulcer and/or bleeding in the remnant stomach, anastomotic stenosis, and gastroesophageal reflux disease. Endoscopic identification of these morbidities allows patients to be treated with appropriate drugs. Since upper gastrointestinal endoscopy can be performed safely now, we believe our recommendations are not extreme and are acceptable.

Conflict of interest This article has no potential and real conflicts of interest.

\section{References}

1. Katsios CS (2010) Metachronous cancer in the stomach remnant [letter]. World J Surg 34. doi:10.1007/s00268-010-0806-8

2. Nozaki I, Nasu J, Kubo Y et al (2010) Risk factors for metachronous gastric cancer in the remnant stomach after early cancer surgery. World J Surg 34:1548-1554

3. Ohashi M, Katai H, Fukagawa T et al (2007) Cancer of the gastric stump following distal gastrectomy for cancer. Br J Surg 94:92-95

4. Hosokawa O, Kaizaki Y, Watanabe K et al (2002) Endoscopic surveillance for gastric remnant cancer after early cancer surgery. Endoscopy 34:469-473
I. Nozaki $(\bowtie) \cdot A$. Kurita

Division of Gastroenterology, Department of Surgery,

National Hospital Organization, Shikoku Cancer Center,

160 Minami-umemoto, Matsuyama 791-0280, Japan

e-mail: isnozaki@shikoku-cc.go.jp 